The Egyptian Journal of Hospital Medicine (January 2019) Vol. 74 (6), Page 1197-1204

\title{
The Value of Late Mid-Trimester Ultrasound Cervical Assessment after Cerclage for the Prediction of Preterm Birth
}

\author{
Yehia Abdul-Rahman El Wasat, Walid Mamdouh Atallah, Ahmed Tawfeek Suliman, Mostafa \\ Zain Al Abideen Mostafa
}

\author{
Department of Obstetrics and Gynecology, Faculty of Medicine, Tanta University, Egypt \\ Corresponding author: Yehia Abdul-Rahman El Wasat, email: dr.yehia86@gmail.com
}

\begin{abstract}
Background: Spontaneous preterm birth constitutes a significant obstetric dilemma as prematurity is a major risk factor of neonatal death or a lifelong morbidity. Despite extensive efforts to reduce preterm birth, the means to identify and prevent it remains limited. Sonographic surveillance of CL can identify the increased risk of spontaneous preterm birth.

Objective: To determine the predictive value of ultrasonographic assessment of the cervix after cerclage insertion for the outcome of preterm birth before 34 weeks of gestation.

Patients and Methods: A prospective study included fifty women with singleton pregnancies were followed-up with monthly transvaginal ultrasonographic examinations before and after cerclage insertion. Ultrasonographic parameters of the cervix included the closed endocervical canal length and funneling depth measured from the internal cervical os.

Results: The change in cervical length was predictive; as with stable cervical length an association with a later gestational age at delivery was evident. Patients with cerclage who delivered after 34 weeks had a slower rate of cervical shortening compared to those who delivered prior to that date (median $=4 \mathrm{~mm}$ versus $7 \mathrm{~mm}$ per 4 weeks). The overall cervical length at 28 weeks was statistically different between patients who delivered before 34 weeks and those who delivered later $(24.00 \pm 2.92$ versus $33.19 \pm 5.5$ $\mathrm{p}<0.001)$.

Conclusions: Transvaginal ultrasound for cervical assessment can be a helpful tool in the prediction of preterm birth after cerclage insertion. A late second-trimester cervical transvaginal ultrasound scan can guide the addition of interventions such, progesterone and antenatal corticosteroids for fetal lung maturation.
\end{abstract}

Keywords: Cerclage, Transvaginal Ultrasound, Preterm birth

\section{Introduction}

Spontaneous preterm birth constitutes a significant obstetric dilemma as prematurity is a major risk factor of neonatal death or a lifelong morbidity. Despite extensive efforts to reduce preterm birth, the means to identify and prevent it remains limited (1). Premature remodeling of the cervix and cervical insufficiency are found to contribute to the etiologies of SPTB ${ }^{(2)}$. Sonographic surveillance of cervical length (CL) can identify the increased risk of spontaneous preterm birth. An inverse relationship exists between cervical length as measured by ultrasound and the risk of SPTB. Moreover, diagnosis of a shortened cervix at before 24 weeks of gestation increases the risk of SPTB (3). Transvaginal ultrasonographic evaluation of the cervix is a safe and well-accepted technique ${ }^{(4,5)}$.
Cervical insufficiency describes the inability of the cervix to retain a pregnancy as a result of painless cervical dilatation leading to subsequent expulsion of the fetus out of the uterus. Cervical insufficiency accounts for 10 $25 \%$ of second trimester pregnancy losses ${ }^{(6)}$. Cervical cerclage is a common procedure that showed to be effective in prevention of midtrimester losses in women at high-risk of cervical insufficiency. Cervical cerclage restores cervical length and the mechanical role of the cervix ${ }^{(6-9)}$. A patient-level meta-analysis of five randomized trials on the effect of cerclage placement in singleton pregnancies with a prior history of preterm delivery demonstrated a significant reduction in recurrent SPTB at $<35$ weeks of $30 \%$, and a $36 \%$ decrease in mortality and morbidity ${ }^{(\mathbf{1 0})}$. 
Cervical funneling can be used as a predictive indicator of preterm delivery. Funneling of fetal membranes to the level of cerclage usually precedes preterm labour and predicts a poor pregnancy outcome ${ }^{(\mathbf{1 1 1}, 12)}$. However, the reliability of funneling as a sole factor for the prediction for preterm birth remains a controversy, as there is no consensus on the technique of funneling measurement ${ }^{(\mathbf{1 3})}$.

However, a woman with cerclage remains at high-risk for preterm delivery as further cervical changes may occur after cerclage. It is confusing to manage progressive cervical shortening after cerclage as the information on prognostic factors in women with cerclage are limited. Corticosteroids administration, admission to high risk antenatal unit, or in-utero transfer to units with appropriate neonatal facilities can be interventional options when progressive cervical shortening is diagnosed in anticipation of preterm delivery ${ }^{(\mathbf{1 4})}$.

Aim of the Work:

The goal of this study is to assess whether cervical length after cerclage influences the pregnancy outcome in women with short CL before cerclage, and to identify the cervical parameters (as the cervical length and funneling depth) related to pregnancy outcome.

\section{Patients and Methods}

This is a prospective study of fifty women with singleton pregnancies who were underwent cervical cerclage placement and had post-cerclage CL assessments at Obstetrics and Gynecology department at Tanta University hospitals during the period from February 2017 to May 2018. After approval of the protocol of the study by the Ethics Committee of Faculty of Medicine, Tanta University, all patients included in the study or their caring relatives were informed about the purpose of the study. The patients were allowed time to consider the study and consult their primary care providers before starting the trial.

The study included both historyindicated and ultrasound-indicated cerclage in the surveillance, rescue cerclage were excluded because they were considered to be at higher risk of preterm rupture of membranes and chorioamnionitis, making cervical length screening less reliable. Pregnancies complicated by major fetal anomalies, severe polyhydramnios, multiple gestations, advanced cervical dilatation, or evident chorioamnionitis were excluded. Demographic data, risk factors for history SPTB, progesterone supplementation, information about previous cerclage placements, presence of cervical tears, delivery data, and serial CL measurements were collected.

Cerclages were placed at 12-14 weeks in the case of a history of two or more preterm deliveries or second trimester losses, or a short cervix less than $25 \mathrm{~mm}$ prior to 24 weeks of gestation in the index pregnancy with a history of preterm labour or preterm rupture of membranes. A McDonald-type purse string cerclage was performed using a permanent nonabsorbable suture (ASTRALEN MERSILENE white tape from Assut Medical Sàrl / Switzerland). Cerclage removal was performed at 36-37 weeks, or when preterm labour was diagnosed. Transvaginal ultrasound imaging was performed via ECM IMAGYNE, (Angoulême, France), 7-9 MHz transvaginal transducer. The cervix was visualized on bladder void; as the bladder falsely elongate the cervix giving false measurements. The probe is gradually withdrawn until the entire cervix is visualized occupying at least $70 \%$ of the scanned area.

Serial monthly assessment of the cervical canal was performed in all patients after the insertion of cerclage at which the shortest functional cervical length from the internal to the external os was recorded each visit. Each transvaginal CL assessment was obtained using the technique published by Perinatal Quality Foundation (15), where the shortest CL for each examination that clearly displayed the internal and external os, of the cervix, demonstrating the opposing anterior and posterior cervix.

Funneling was defined as the prolapse of fetal membranes into the cervical canal. Funneling of the membranes beyond the internal os was evaluated at rest or in response to fundal pressure. Funnel depth more than 5 $\mathrm{mm}$ was considered significant. The progression of changes at the level of the internal os from a "T" to a "V" to a "U" shape 
was documented. Funneling depth was recorded in all patients if present.

The exposure of interest in this study was association of cervical shortening after cerclage insertion and the risk of SPTB. SPTB was defined as delivery prior to 34 weeks, which delivery before 34 weeks of gestation is associated with a significant increase in neonatal mortality and morbidity. All statistical analyses were performed using SPSS software version 22.

\section{Results}

Fifty patients (all singleton pregnancies) were included in this study; the mean gestational age \pm SD at delivery for the entire cohort was $35.9 \pm 2.3$ weeks. The gestational age at delivery was significantly different between two groups. Patients were divided into two groups according to delivery at 34 weeks of gestation. One group delivered before 34 weeks of gestation $(18 \%, n=9)$ and the other group delivered after 34 weeks of gestation $(82 \%, \mathrm{n}=41)$. The mean gestational age \pm SD at delivery for the two groups was $32.1 \pm 2.5,36.8 \pm 1.1$ respectively. There were no significant demographic differences between the two groups. The demographic characteristics of the study population are shown in Table I. The mean immediate increase in cervical length was $9.2 \mathrm{~mm}$ in patients who delivered before 34 weeks as compared with 11 $\mathrm{mm}$ in patients who delivered after 34 weeks. Figure II shows cervical length measurements among the studied groups. Patients who delivered after 34 weeks had a higher accelerated rate of shortening at the period between 24 - 28 weeks of gestation and shorter cervical length measurements. Women that delivered before 34 weeks had significantly higher rates of cervical funneling compared to those who delivered later $(66.6 \%$ vs. $19.5 \%$, P $=0.004)$. Funneling depth was statistically significant between the women who delivered before 34 weeks and those who delivered later (9.77 \pm 6.64 vs. $3.68 \pm 2.04, p<0.001)$.

Table I: Demographic characteristics of the study population.

\begin{tabular}{llll}
\hline Variable & $\begin{array}{l}\text { Delivery <34 } \\
\text { wks.(n=9) }\end{array}$ & $\begin{array}{l}\text { Delivery } \geq \mathbf{3 4} \\
\text { wks.(n=41) }\end{array}$ & p-value \\
\hline Maternal age (Mean + SD) & $23.88 \pm 2.42$ & $25.2 \pm 3.13$ & 0.238 \\
Gravidity (Median (Range)) & $3(2-6)$ & $4(2-7)$ & 0.239 \\
Parity (Median (Range)) & $0(0-1)$ & $1(0-3)$ & 0.124 \\
Previous mid-trimester losses (Median, Range) & $2(1-4)$ & $2(1-5)$ & 0.691 \\
Previous cerclage $(\%)$ & $4(44.4 \%)$ & $26(63.4 \%)$ & $0.004^{*}$ \\
Presence of cervical tears (\%) & $1(11.1 \%)$ & $4(9.8 \%)$ & 0.902 \\
Gestational age at delivery (Mean $\mathbf{\pm}$ SD) & $32.1 \pm 2.5$ & $36.8 \pm 1.1$ & $0.006^{*}$ \\
\hline n= Number SD= Standard deviation & & &
\end{tabular}

Table II: Cervical measurements of the study population.

\begin{tabular}{|c|c|c|c|}
\hline Variable & $\begin{array}{l}\text { Delivery <34 wks. } \\
(n=9)\end{array}$ & $\begin{array}{l}\text { Delivery } \geq 34 \text { wks. } \\
(n=41)\end{array}$ & p-value \\
\hline Preoperative CL & $21.66 \pm 4.06$ & $25.17 \pm 3.95$ & $0.021 *$ \\
\hline Post-operative CL & $29.88 \pm 3.37$ & $36.09 \pm 4.04$ & $<0.001 * *$ \\
\hline Difference & $9.22 \pm 3.11$ & $11.09 \pm 3.60$ & 0.155 \\
\hline CL at 20 weeks & $28.40 \pm 3.88$ & $35.56 \pm 4.76$ & $<0.001 * *$ \\
\hline CL at 24 weeks & $28.22 \pm 2.86$ & $35.63 \pm 4.60$ & $<0.001 * *$ \\
\hline CL at 28 weeks & $24.00 \pm 2.92$ & $33.19 \pm 5.54$ & $<0.001 * *$ \\
\hline Funnel depth at 28 weeks (mm) & $9.77 \pm 6.64$ & $3.68 \pm 2.04$ & $<0.001 * *$ \\
\hline CL at 32 weeks & $23.28 \pm 2.69$ & $31.73 \pm 5.23$ & $<0.001 * *$ \\
\hline Rate of change in length/week (Median, Range) & $-7(-12 /-3)$ & $-4(-13 /-2)$ & $0.043 *$ \\
\hline
\end{tabular}




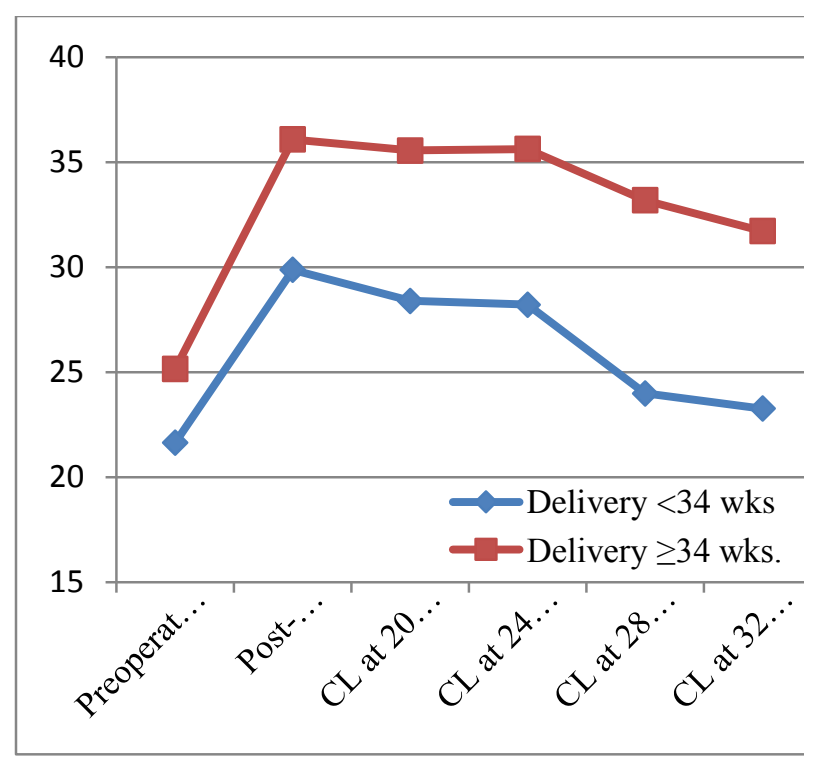

Figure I: Cervical length measurements among the studied groups.

\section{ROC Curve}

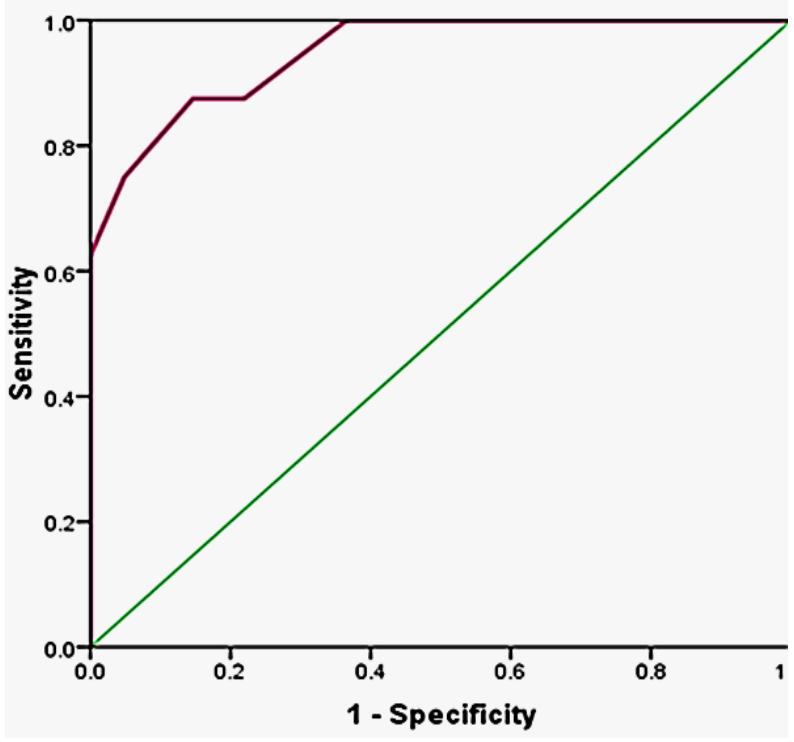

Figure II: Roc curve for prediction of preterm delivery $<34$ weeks by cervical length $<25 \mathrm{~mm}$ at 28 weeks (area under curve, 0.95; 95\% confidence interval, 0.873 - 1.024; positive predictive value: $75 \%$ ). The timing at 28 weeks was chosen for the statistical differences between the studied groups.

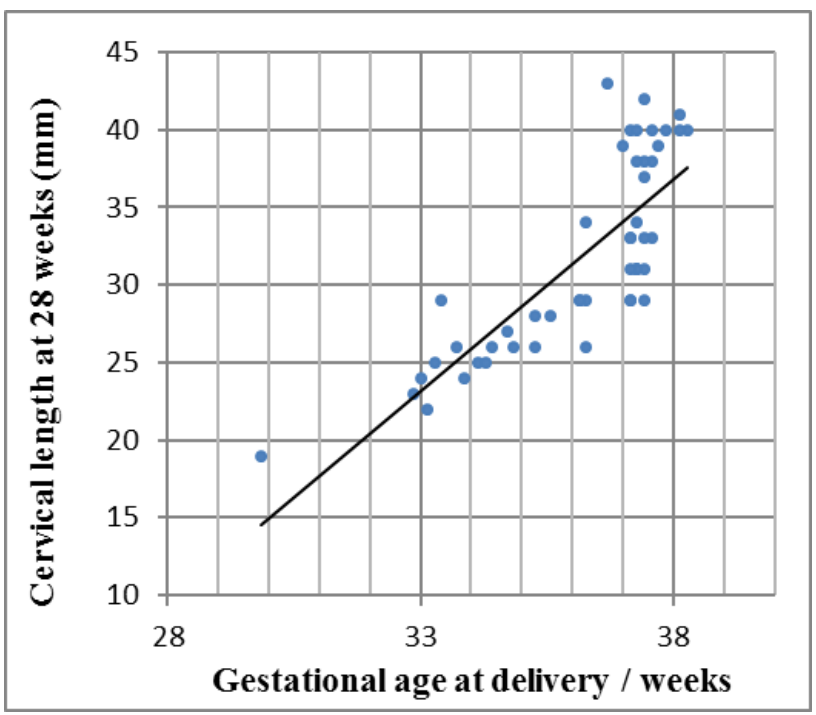

Figure III: Scatter diagram for correlation between gestational age at delivery and cervical length at 28 weeks showing high correlation between cervical length at 28 weeks and gestational age at delivery.

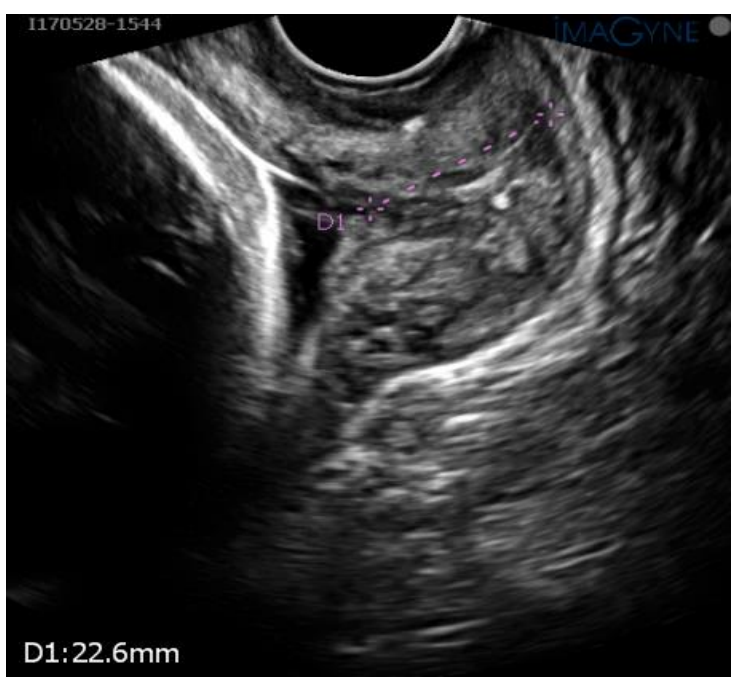

Figure IV: a 21-year-old G2P0A1 with ultrasound-indicated cerclage. TVU at 30 weeks of gestation showing progressive shortening that lasted over the second trimester. Tocolytics and corticosteroids were administered at 28 weeks. The patient delivered at 33 weeks. 


\section{Discussion}

The majority of neonatal mortality and morbidity is caused by preterm birth. Cervical cerclage has an important beneficial effect in a minority of pregnant women, those who suffer from cervical insufficiency. The value of ultrasound as a diagnostic tool for cervical insufficiency has reshaped the use of cervical cerclage. However, the need for more predictive findings urges researchers to determine further abilities of serial ultrasonography after cerclage insertion that may be useful in predicting outcomes ${ }^{(16)}$.

In the present study, the majority of patients delivered on average near term, when neonatal morbidity would be expected to be low. The mean gestational age at delivery for the entire cohort was 35.9 weeks. Patients were divided into two groups according to delivery at 34 weeks of gestation as it was chosen as a cutoff point because of the decreased morbidity and mortality after this point ${ }^{(\mathbf{1 6})}$.

Nevertheless, there was a significant increase in overall cervical length after cerclage insertion. The mean increase in cervical length was $9.2 \mathrm{~mm}$ in patients who delivered before 34 weeks as compared with $11 \mathrm{~mm}$ in patients who delivered after 34 weeks. However, the degree of cervical length increment after cerclage did not contribute to the odds of delivery after 34 weeks. This finding is consistent with other studies, which found an immediate increase in cervical length after cerclage was not predictive of term delivery ${ }^{(\mathbf{1 7}, 18)}$.

The results of the present study find some contrast with other studies which found the immediate increase post procedure is suggestive of delivery after 34 weeks. This could be due to the inclusion of rescue cerclage cases in one study which can confuse the results ${ }^{(19)}$, and the sole nature of ultrasound-indicated cerclage with cervical length less than $25 \mathrm{~mm}$ in another study ${ }^{(20)}$.

\section{Rate of cervical shortening}

In both groups of patients, there was a progressive decrease in cervical length in patients who delivered before 34 weeks and in those who delivered after 34 weeks of gestation. However, patients who delivered after 34 weeks retained stable cervical length for a longer duration. They showed a slower rate in length changes over time compared with patients who delivered prior to that date.

Monthly ultrasonographic examinations showed that the increased shortening of the cervix between 24 to 28 weeks resulted in an increased risk of delivery before 34 weeks in contrast with women whose cervix remained relatively constant after cerclage insertion. Furthermore, there was a statistically significant difference across gestational weeks in mean cervical length at (24-28 weeks) in patients who delivered before 34 weeks compared with those delivered later.

In this study, we found a correlation between cervical length near the end of second trimester at 24-28 weeks and preterm delivery before 34 weeks of gestation. As the functional cervical length got longer, the probability of preterm delivery before 34 weeks of gestation was reduced. The ROC curve had an area under the curve of 0.95 with a 95 percent confidence interval of 0.87-1.02 when $25 \mathrm{~mm}$ was used as a cutoff for functional cervical length at 28 weeks of gestation. It is reasonable that, a cervical length of $25 \mathrm{~mm}$ measured at 24-28 weeks can be used as a baseline reference for early preterm delivery before 34 weeks of gestation.

Both short cervical length less than 25 $\mathrm{mm}$ and an accelerated rate of cervical shortening carry an increased risk of delivery before 34 weeks in patients with a cerclage in situ. Drassinower et al. found that those who had higher rate of shortening after the cerclage delivered earlier as compared with patients with stable cervical length postoperatively ${ }^{(14)}$.

We included both history-indicated and ultrasound-indicated cerclages in this study, the rates of cervical shortening were found to be similar whether the indication for cerclage. This 
finding is consistent with other studies who found that the success rate of cerclage is not dependent on cervical length before cerclage

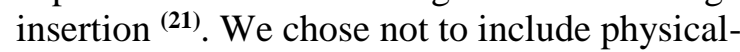
exam indicated cerclage in this study as it is known that; the presence of visible fetal membranes at the external os at the time of surgery compromises a potential risk of preterm rupture of membranes and chorioamnionitis. A cervical length less than $15 \mathrm{~mm}$ at the time of cerclage insertion with visible membranes is usually associated with poor pregnancy outcome $^{(22)}$.

\section{Funneling of Fetal Membranes}

The current findings suggest that funneling in post-cerclage patients is associated with poor pregnancy outcome. Furthermore, funneling depth at 20-28 weeks is highly predictive of preterm delivery, thus suggesting that this finding is helpful in anticipating preterm birth. We found that funneling of fetal membranes after cerclage placement in the late second / early third trimester correlated with preterm delivery before 34 weeks because of preterm premature rupture of membranes as seen in 3 pregnancies that were noted to have funneling after cerclage insertion.

Unlike CL, the technique of funneling measurement is various among practitioners. In this study, we defined funneling as funneling depth of more than $5 \mathrm{~mm}$. Other methods commonly used include examining the funneling shape $(\mathrm{T}, \mathrm{V}, \mathrm{U})$, and the cervical score (CL minus cervical dilatation). 3D Ultrasound may be a more accurate quantification as compared to a conventional 2D US ${ }^{(13)}$.

Our results demonstrated that pregnancies that delivered before 34 weeks recorded significantly greater rates of cervical funneling as compared to those who delivered later $(66.6 \%$ vs. $19.5 \%, \mathrm{P}=0.004)$. This result is consistent with others who reported that the presence of funneling significantly increased the chance of preterm delivery. O'Brien $\boldsymbol{e t}$ al. (11) reported that funneling at the cerclage stitch level significantly contribute to premature rupture of membrane and preterm labour. Dijkstra et al. (18) further found that the presence of a measurable cervix above the cerclage in both history-indicated and ultrasound-indicated cases increases the likelihood of term delivery. Other studies that have noted the funneling can be due to possible infection or stromal weakening and reflected a different concern than just cervical shortening (23).

\section{Serial screening versus a single transvaginal ultrasound scan}

The timing for cervical assessment by ultrasound vary widely among different studies, the recommended frequency of serial transvaginal ultrasound examinations differs from weekly to monthly until 28-32 weeks $(11,14,18)$.

Although serial evaluation of cervical length seems to be a valuable tool in determining the risk of preterm delivery, it might not be as useful a marker in patients with cerclage until near the end of second trimester of pregnancy. We find that the length of cervix after cerclage at 24-28 weeks can be used as moderately confident predictor of preterm delivery. This study supports several other studies that observed a progressive shortening of the cervix toward early the third trimester when transvaginal ultrasound examinations was performed in both history and ultrasoundindicated cerclage insertions ${ }^{(\mathbf{1 7}, \mathbf{2 4})}$.

\section{Conclusions}

The current study concluded that; progressive cervical shortening and funneling would be used as predictors of preterm birth in patients with cervical insufficiency who had undergone cervical cerclage and post-operative follow-up by transvaginal ultrasonography, these changes are remarkably observed near the end of the second trimester. A cervical length less than $25 \mathrm{~mm}$ after cerclage at the end of the second trimester (24-28 weeks) is highly predictive of preterm delivery before 34 weeks.

\section{References}


1. Blencowe H, Cousens S, Chou D et al. (2013): Born too soon: the global epidemiology of 15 million preterm births. Reproductive Health, 10(1): 216.

2. Word RA, Li XH, Hnat M et al. (2007): Dynamics of cervical remodeling during pregnancy and parturition: mechanisms and current concepts. Seminars in Reproductive Medicine, 25(01): 69-79.

3. Heath VC, Southall TR (1998): Cervical length at 23 weeks of gestation: prediction of spontaneous preterm delivery. Ultrasound in Obstetrics and Gynecology. The Official Journal of the International Society of Ultrasound in Obstetrics and Gynecology, 12(5): 312-317.

4. Kagan KO, Sonek J (2015): How to measure cervical length. Ultrasound in Obstetrics \& Gynecology, 45(3): 358362.

5. Clement $S$, Candy $B$, Heath $\mathrm{V}$ et al. (2003): Transvaginal ultrasound in pregnancy: its acceptability to women and maternal psychological morbidity. Ultrasound in Obstetrics and Gynecology, 22(5): 508-514.

6. American College of Obstetricians and Gynecologists (2014): Cerclage for the management of cervical insufficiency. Obstetrics and Gynecology, 123(2): 372-375.

7. Shennan AH, To MS (2011): Greentop guideline No 60: cervical cerclage. https://www.rcog.org.uk/en/guidelines -research-services/guidelines/gtg60/

8. O'Connell MP, Lindow SW (2001): Reversal of asymptomatic cervical length shortening with cervical cerclage: a preliminary study. Human Reproduction, 16(1): 172-173.

9. Owen J, Hankins G, Iams JD et al. (2009): Multicenter randomized trial of cerclage for preterm birth prevention in high-risk women with shortened midtrimester cervical length. American Journal of Obstetrics and Gynecology, 201(4): 375-377.
10. Berghella V, Odibo A, To $M$ et al. (2005): Cerclage for short cervix on ultrasonography: meta-analysis of trials using individual patient-level data. Obstetrics \& Gynecology, 106(1): 181189.

11. O'brien JM, Hill AL and Barton JR (2002): Funneling to the stitch: an informative ultrasonographic finding after cervical cerclage. Ultrasound in Obstetrics \& Gynecology, 20(3): 252255.

12. Miller ES, Gerber SE (2014): Association between sonographic cervical appearance and preterm delivery after a history-indicated cerclage. Journal of Ultrasound in Medicine, 33(12): 2181-2186.

13. Song JE, Lee KY, Kim MY et al. (2012). Cervical funneling after cerclage in cervical incompetence as a predictor of pregnancy outcome. The Journal of Maternal-Fetal Neonatal Medicine, 25(2): 147-150.

14. Drassinower D, Vink J, Pessel C et al. (2015): Effect of cervical cerclage on rate of cervical shortening. Ultrasound in Obstetrics \& Gynecology, 46(6): 718-723.

15. Khalifeh A, Berghella V (2016): Universal cervical length screening in singleton gestations without a previous preterm birth: ten reasons why it should be implemented. American Journal of Obstetrics and Gynecology, 214(5): 603-606.

16. Marret S, Ancel PY, Marpeau L et al. (2007): Neonatal and 5-year outcomes after birth at 30-34 weeks of gestation. Obstetrics \& Gynecology, 110(1): 7280.

17. Sim S, Da Silva C (2015): Factors associated with spontaneous preterm birth risk assessed by transvaginal ultrasound following cervical cerclage. Australian and New Zealand Journal of Obstetrics and Gynaecology, 55(4): 344-349.

18. Dijkstra K, Funai EF, O'Neill J et al. (2000): Change in cervical length after cerclage as a predictor of preterm 
delivery. Obstetrics \& Gynecology, 96(3): 346-350.

19. Song RK, Cha HH, Shin MY et al. (2016): Post-cerclage ultrasonographic cervical length can predict preterm delivery in elective cervical cerclage patients. Obstetrics \& Gynecology Science, 59(1):17-23.

20. Cook JR, Chatfield S, Chandiramani M et al. (2017): Cerclage position, cervical length and preterm delivery in women undergoing ultrasound indicated cervical cerclage: A retrospective cohort study. PloS One, 12(6): 17-72.

21. Berghella V, Keeler SM, To MS et al. (2010): Effectiveness of cerclage according to severity of cervical length shortening: a meta-analysis. Ultrasound in Obstetrics and Gynecology: The Official Journal of the International Society of Ultrasound in Obstetrics and Gynecology, 35(4): 468-473.

22. Terkildsen MC, Parilla BV, Kumar $P$ et al. (2003): Factors associated with success of emergent second-trimester cerclage. Obstetrics \& Gynecology, 101(3): 565-569.

23. Berghella V, Owen J, MacPherson D et al. (2007): Natural history of cervical funneling in women at high risk for spontaneous preterm birth. Obstetrics \& Gynecology, 109(4): 863-869.

24. Hedriana H, Lanouette J, Haesslein H et al. (2008): Is there value for serial ultrasonographic assessment of cervical lengths after a cerclage?. American Journal of Obstetrics and Gynecology, 198(6): 705-707. 